\title{
Polymerization Shrinkage and Flexural Modulus of Flowable Dental Composites
}

\author{
Janaína Cavalcanti Xavier ${ }^{\mathrm{a}}$, Gabriela Queiroz de Melo Monteiro ${ }^{\mathrm{b}, *}$, Marcos Antonio Japiassú Resende Montes ${ }^{\mathrm{a}}$ \\ ${ }^{\mathrm{a}}$ Departamento de Odontologia Restauradora, \\ Faculdade de Odontologia - FOP, Universidade de Pernanbuco - UPE, \\ Camaragibe, PE, Brazil \\ ${ }^{\mathrm{b}}$ Curso de Odontologia, Centro Universitário de João Pessoa - UNIPÊ, João Pessoa, PB, Brazil
}

Received: March 7, 2010; Revised: June 13, 2010

\begin{abstract}
Linear polymerization shrinkage (LPS), flexural strength (FS) and modulus of elasticity (ME) of low-viscosity resin composites (Admira Flow ${ }^{\mathrm{TM}}$, Grandio Flow ${ }^{\mathrm{TM}} /$ VOCO; Filtek Z350 Flow ${ }^{\mathrm{TM}} / 3 \mathrm{M}$ ESPE; Tetric Flow ${ }^{\mathrm{TM}} / \mathrm{Ivoclar}^{-}$ Vivadent) was evaluated using a well-established conventional micro-hybrid composite as a standard (Filtek Z250 $2 \mathrm{M} / 3 \mathrm{M}$ ESPE). For the measurement of LPS, composites were applied to a cylindrical metallic mould and polymerized $(n=8)$. The gap formed at the resin/mould interface was observed using SEM $(1500 \times)$. For FS and ME, specimens were prepared according to the ISO 4049 specifications $(n=10)$. Statistical analysis of the data was performed with one-way ANOVA and the Tukey test. The conventional resin presented significantly lower LPS associated with high FS and ME, but only the ME values of the conventional resin differed significantly from the low-viscosity composites. The relationship between ME and LPS of low-viscosity resin composites when used as restorative material is a critical factor in contraction stress relief and marginal leakage.
\end{abstract}

Keywords: composite resins, flexural strength, flowable composites, modulus of elasticity, polymerization shrinkage

\section{Introduction}

Since the development of resin-based composites, these materials have undergone many changes towards ideal mechanical and clinical characteristics. Despite these improvements, overcoming polymerization shrinkage remains one of the main challenges.

Polymerization shrinkage of resin-based materials promotes deformation of the material. In clinical situations, these materials are bonded to cavity walls in such way that this deformation is restricted, leading to the development of stress. These stresses are concentrated at the adhesive interface, and can cause disruption of the interface and consequently gap formation, marginal leakage and discoloration, post-operative sensitivity and recurrent caries ${ }^{1}$.

Some techniques have been proposed to minimize this problem, such as the application of an elastic intermediate layer (or liner) between the adhesive layer and the non-flowable/conventional composite material, fulfilling the concept of an 'elastic cavity wall'. The idea behind this is that the shrinkage stress of subsequently applied resin composite can be absorbed by a relatively elastic initial layer, thereby reducing the stress at the restorative-tooth interface. Stress absorption is a function of thickness and modulus, and, for any given modulus, a thicker layer will absorb more stress ${ }^{2-4}$.

Materials proposed for the application of this concept include unfilled resins and flowable composites. Flowable composites are low-viscosity resin-based restorative materials that differ from conventional resin composites in their filler load and resin content. Flowables contains the same filler particles as traditional hybrid composites but contains $20-25 \%$ less filler than non-flowable/ conventional materials ${ }^{5}$. The reduction in the filler concentration has a direct influence on the modulus of elasticity of these materials. The increase in the resin content and the large amount of diluent monomers, such as tetraethylene glycol dimethacrylate (TEGDMA), gives the material its flow characteristics. Thus, flowable materials have two desirable characteristics, non-stickiness and fluid injectability, which allow them not only to fulfil the elastic cavity wall concept (as a liner), but also to restore micro-conservative occlusal cavities, class III, IV and V restorations, tunnel preparation restoration, restorations repairs, pit fissure sealants and cementing translucent restorations ${ }^{6}$.

According to Hooke's law, stress is determined by the stiffness of the material when subjected to a given strain. Therefore, the higher the elastic modulus and/or the polymerization shrinkage of the composite, the higher the contraction stress will be. These two factors seem to act synergistically ${ }^{7}$.

Based on the required characteristics, the composition of the material will have a major influence on its final properties. For this reason, the purpose of this study was to evaluate the linear polymerization shrinkage (LPS), the flexural strength (FS) and modulus of elasticity (ME) of low-viscosity resin composites, using a well-established conventional micro-hybrid composite as a standard. The null hypothesis tested was that differences in composition do not affect LPS and ME among selected materials.

\section{Materials and Methods}

The materials, manufacturers, composition and batch numbers for this study are listed in Table 1.

\subsection{Linear polymerization shrinkage}

The restorative composites were placed in a circular metallic mould (inner diameter $7 \mathrm{~mm}$, height $2 \mathrm{~mm}$ ). The composites were then covered with a Mylar strip and pressed with a microscope glass slab. Eight specimens were prepared for each material. Photo-activation was performed with the curing tip positioned close to the metallic mould/restorative composite for 40 seconds, with a minimum light intensity of $500 \mathrm{~mW} \cdot \mathrm{cm}^{-2}$ using an Optilight Plus ${ }^{\mathrm{TM}}$ curing unit (Gnatus, Ribeirão Preto, São Paulo, Brazil). The light intensity was measured 
Table 1. Composition, manufacturer and batch number of the materials.

\begin{tabular}{|c|c|c|c|}
\hline Composite & Matrix $^{a}$ & $\begin{array}{l}\text { Filler content; filler size; } \\
\% \text { by mass/volume }\end{array}$ & $\begin{array}{l}\text { Manufacturerb; } \\
\text { batch number }\end{array}$ \\
\hline Filtek Z250 & BisGMA, UDMA and BisEMA & Zirconia/silica; micro-hybrid; 82/60 (8) & 3M/ESPE; 5WK \\
\hline Admira Flow ${ }^{\mathrm{TM}}$ & Anorganic-organic co-polymers & Ormocer; $-; 64 / 55.5$ & VOCO; 651555 \\
\hline Filtek Z350 Flow ${ }^{\mathrm{TM}}$ & BisGMA, TEGDMA and BisEMA & $\begin{array}{l}\text { Zirconia/silica and silica; } \\
\text { nanoparticle; } 65 / 55\end{array}$ & 3M/ESPE; $5 \mathrm{AK}$ \\
\hline Grandio Flow ${ }^{\mathrm{TM}}$ & BisGMA, TEGDMA and HEDMA & Glass ceramic; nanoparticle; 80/65.6 & VOCO; 650823 \\
\hline Tetric Flow ${ }^{\mathrm{TM}}$ & BisGMA, TEGDMA and UDMA & $\begin{array}{l}\text { Barium glass, ytterbium trifluoride; } \\
\text { microhybrid; } 64.6 / 39.7\end{array}$ & $\begin{array}{c}\text { IVOCLAR/ } \\
\text { VIVADENT; } 60885\end{array}$ \\
\hline
\end{tabular}

All composites were A3 color. -, information not available from the manufacturer.

aBisGMA, bisphenol-glycidyl methacrylate; TEGDMA, triethylene glycol dimethacrylate; BisEMA, bisphenol-polyethylene glycol dimethacrylate; HEDMA, hydroxyethyl dimethacrylate; UDMA, urethanethyl dimethacrylate.

${ }^{b}$ VOCO, Cuxhaven, Germany; 3M ESPE, St. Paul, MN, USA; IVOCLAR/VIVADENT, Schaan, Liechtenstein.

with a radiometer (Gnatus, Ribeirão Preto, São Paulo, Brazil). After 15 $\min$, the top and bottom surfaces of the specimens were polished with sandpaper of decreasing grit (nos. 320, 600, 1200) and then placed in an ultrasound cube for 2 minutes. After 24 hours at $37^{\circ} \mathrm{C}$, the specimens were mounted on stubs, gold sputtered (sputter coater SCD 050 Bal-Tec, Schaan, Liechtenstein) and, using scanning electron microscopy (JEOL JSM-6360 SEM, Japan; 1500×), the gap formed between the metallic mould and the resin composite was observed at 4 points located in positions corresponding to 3, 6,9 and 12 hours of a clock face $^{8}$. Images were taken and the gaps were measured (in micrometers) using the Image J program (Image Processing and Analysis in Java) ${ }^{9}$. The arithmetic means were calculated for each specimen.

\subsection{Flexural strength and modulus of elasticity}

FS was determined according to the International Organization for Standardization (ISO) Standard 4049 ${ }^{[10]}$. Resin composites were inserted in a split stainless steel mould $(25 \times 2 \times 2 \mathrm{~mm})$, covered with a Mylar strip and a glass microscope slab, then light-cured using the Optilight Plus curing unit described above. The light curing procedure was performed with three overlapping sections of 40 seconds each. This procedure was repeated on the opposite side. Ten specimens were prepared for each group. Fifteen minutes after light curing, the specimens were then freed from the mould, gently wet-ground (sandpaper paper no. 320) to remove any flash, and transferred to a distilled water bath at $37{ }^{\circ} \mathrm{C}$. After 24 hours, the dimensions of the specimens were measured to an accuracy of $0.01 \mathrm{~mm}$ using a digital micrometer. The three-point bend fixture consisted of two rods (diameter $2 \mathrm{~mm}$ ) mounted parallel with $20 \mathrm{~mm}$ between their centres (support span $20 \mathrm{~mm}$ ). Each specimen was loaded at its centre with a $2-\mathrm{mm}$ diameter striker at a cross-head speed of $0.5 \mathrm{~mm} / \mathrm{min}$ until failure (Universal Testing Machine, Kratos Dynamometers, São Paulo, Brazil). Flexural strength $(\sigma)$ and modulus of elasticity $(E)$ were calculated using the Equations 1 and 2:

$$
\begin{aligned}
& \sigma=3 P L / 2 b h^{2}(\text { measured in } \mathrm{MPa}) \\
& E=F L^{3} / 4 b h^{3} d \times 10^{-3}(\text { measured in } \mathrm{GPa})
\end{aligned}
$$

where $L$ is the distance between the supports (fixed at $20 \mathrm{~mm}$ ), $b$ is the specimen width $(\mathrm{mm}), h$ is the specimen height $(\mathrm{mm}), F$ is the load $(\mathrm{N})$ at a convenient point on the straight line portion of the curve, $d$ is the deflection $(\mathrm{mm})$ at load $F$, and $P$ is the maximum load (N) resulting in failure.

Statistical evaluation of the data was performed by one-way analysis of variance (ANOVA) and the Tukey test; a 5\% significance level was used $(p=0.05)$.

\section{Results}

The results for the LPS are shown in Figure 1. ANOVA rejected the null hypothesis, showing that at least one of the groups differed from the rest. The Tukey paired comparisons test, at 5\% $(p<0.05)$, demonstrated statistically significant differences between Filtek $\mathrm{Z}_{250^{\mathrm{TM}}}$ and all the other groups except for Tetric flow ${ }^{\mathrm{TM}}$. Admira Flow $^{\mathrm{TM}}$, Grandio Flow ${ }^{\mathrm{TM}}$ and Filtek Z350 Flow $^{\mathrm{TM}}$ had comparable values, as shown by the different letters in parentheses.

ANOVA also rejected the null hypothesis for the FS (Figure 2), and the results varied from $88.94 \mathrm{MPa}$ (Admira Flow ${ }^{\mathrm{TM}}$ ) to 126.87 MPa (Filtek Z250 ${ }^{\mathrm{TM}}$ ), with significant differences existing between these groups $(p<0.05)$. It was interesting to observe that Tetric Flow ${ }^{\mathrm{TM}}$ and Gandio Flow ${ }^{\mathrm{TM}}$ did not differ from the results obtained by Filtek Z250 ${ }^{\mathrm{TM}}$.

Results for the ME are shown in Figure 3. Filtek Z250 ${ }^{\mathrm{TM}}$ presented the highest mean values ( $8.52 \mathrm{GPa})$. Although statistically different from the conventional resin, Grandio Flow $^{\mathrm{TM}}$ presented higher modulus of elasticity $(6.85 \mathrm{GPa})$ between all the low-viscosity composites evaluated.

\section{Discussion}

\subsection{Linear polymerization shrinkage}

The results for the LPS can be related to the diverse compositions of the studied resins. Each of the composites has at least one unique monomer, that is, it is the only composite with that monomer. For example, Tetric Flow ${ }^{\mathrm{TM}}$ contains urethane dimethacrylate (UDMA), Filtek Z350 flow $^{\mathrm{TM}}$ contains bisphenolpolyethylene glycol dimethacrylate (BisEMA), Grandio Flow ${ }^{\mathrm{TM}}$ contains hydroxyethyl dimethacrylate (HEDMA) and Admira Flow $^{\mathrm{TM}}$ contains anorganic-organic copolymers. Apart from Admira Flow ${ }^{\mathrm{TM}}$, all the low-viscosity resins contain TEGDMA. The presence of this diluent monomer favours the reduction in viscosity that characterizes the material. On the other hand, higher concentrations of these monomers have a negative effect on polymerization shrinkage because of the smaller size of the molecule. ${ }^{11}$ Filtek $\mathrm{Z} 250^{\mathrm{TM}}$, the conventional resin, does not have a "unique" monomer. It contains a mixture of BisGMA, UDMA and BisEMA all of which are high molecular weight monomers with high viscosity and low polymerization shrinkage (Table 1).

Admira Flow ${ }^{\mathrm{TM}}$ is classified as an Ormocer. The Ormocer structure comprises an inorganic/organic network formed by polycondensation. Unlike conventional polymers, Ormocers have an inorganic backbone based on $\mathrm{SiO}_{2}$ and are functionalized with polymerizable organic units 


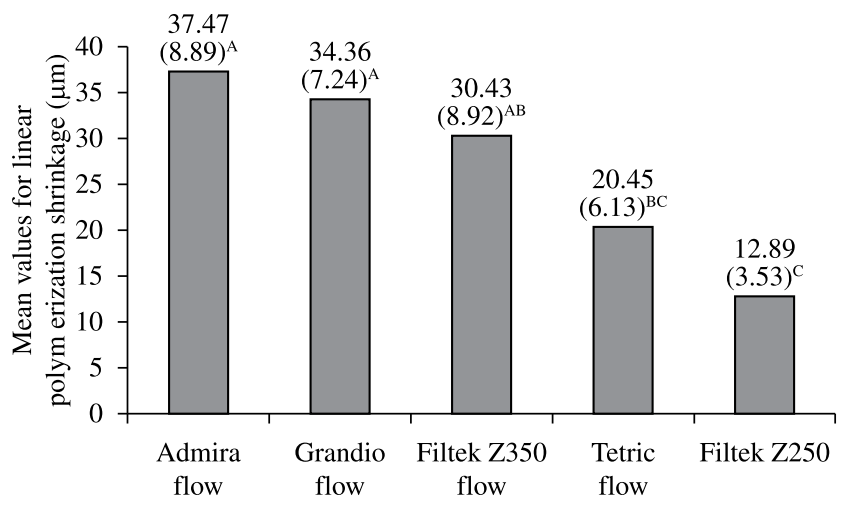

Figure 1. Mean values of LPS $(\mu \mathrm{m})$ and standard deviations ( ) of the materials studied. Different superscript letters indicates that the materials are statistically different at $5 \%$ using the Tukey test.

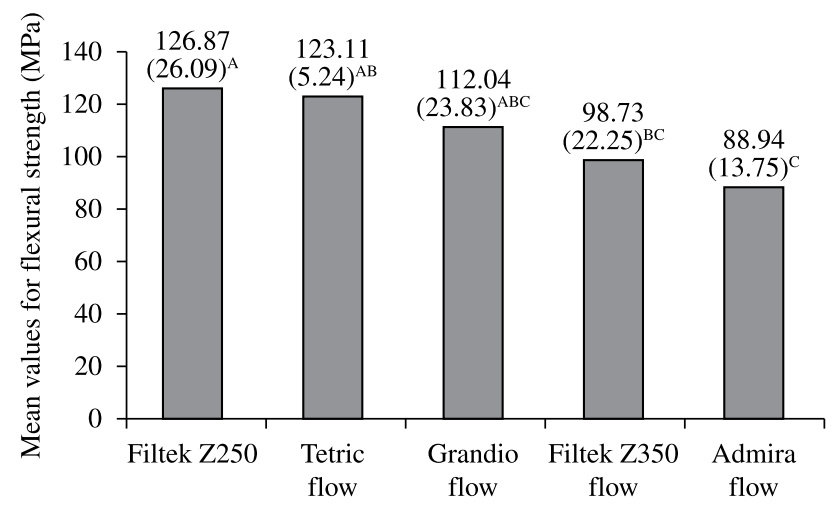

Figure 2. Mean values of FS (MPa) and standard deviations ( ) of the materials studied. Different superscript letters indicates that the materials are statistically different at $5 \%$ using the Tukey test.

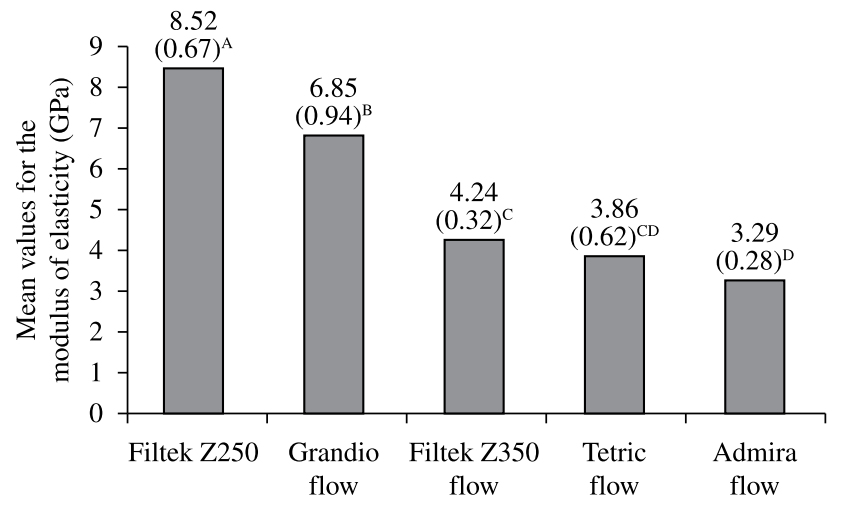

Figure 3. Mean values of ME (GPa) and standard deviations ( ) of the materials studied. Different superscript letters indicates that the materials are statistically different at 5\% using the Tukey test.

to produce three-dimensional compound polymers. Filler particles are also incorporated into this cross-linked inorganic-organic network, mainly made up of special glass ceramic and highly dispersed silica. The main advantages claimed by the manufacturer are its improved biocompatibility and mechanical properties ${ }^{12,13}$.
Filler concentration also has an influence on polymerization shrinkage, since it leads, to a certain extent, to a reduction in monomer concentration. A high filler concentration also reduces the viscosity of composites, which justifies the smaller concentrations of the flowable composites compared with a conventional resin. The only exception is Grandio Flow ${ }^{\mathrm{TM}}$, which has a higher filler concentration by volume $(65.6 \%)$ than Filtek Z250 ${ }^{\mathrm{TM}}(60 \%)$. Nonetheless, this composite showed high polymerization shrinkage, probably due to the presence of two diluent monomers, TEGDMA and hexaethylene glycol dimethacrylate (HEGDMA), which reinforces the greater importance that the monomer composition has on polymerization shrinkage.

The LPS for Tetric Flow ${ }^{\mathrm{TM}}$, although numerically different from that of Filtek $\mathrm{Z} 250^{\mathrm{TM}}$, was not statistically significant. Analyzing the composition of these composites, there is a significant reduction in filler concentration by volume for the flowable composite $(39.7 \%)$. The monomer composition differed only in the presence of TEGDMA. In this case, the concentration of the diluent monomer is probably very low, and does not interfere with polymerization shrinkage, in accordance with other results ${ }^{5}$.

\subsection{Flexural strength test}

FS was measured as a method of calculating the modulus of elasticity. The modifications in the materials formulations to give its flow characteristics also affected the mechanical properties.

Flowable composites are indicated not only as an intermediate layer, but also as a restorative material for minimally invasive cavities, fissure sealing and for resin composite restoration repair. For those indications, specific mechanical properties are required. Analyzing results from an FS test is an acceptable way to predict the behaviour of the material submitted to masticatory forces. Only Tetric Flow ${ }^{\mathrm{TM}}$ and Grandio Flow ${ }^{\mathrm{TM}}$ showed similar results when compared with the conventional composite.

Studies on the influence of monomer composition on the mechanical properties of resin composites have found that FS increases when BisGMA or TEGDMA are substituted by UDMA, as in Tetric Flow ${ }^{\mathrm{TM}}$. Other factors such as filler size, composition and concentration, the amount of initiators and the quality of silanization can also contribute to the development of physical and mechanical properties $^{11}$.

The high FS values exhibited by Grandio Flow ${ }^{\mathrm{TM}}$ could be attributed to the higher filler concentration. This high concentration favours the development of mechanical properties such as compressive strength, hardness, flexural strength and modulus of elasticity ${ }^{6,14}$; however, such a direct relationship between filler concentration and FS has not been demonstrated in this work (i.e. Tetric Flow ${ }^{\mathrm{TM}}$ ) and confirmed by other authors ${ }^{15,16}$.

\subsection{Modulus of elasticity}

The results for ME demonstrated statistically significant differences between the conventional resin (Filtek Z250 ${ }^{\mathrm{TM}}$ ) and the other flowable resins; Filtek Z250 ${ }^{\mathrm{TM}}$ had the highest value (8.52 GPa). This is in line with the clinical applications of the materials. Amongst the low-viscosity composites, Grandio Flow ${ }^{\mathrm{TM}}$ had statistically significant higher ME (6.85 GPa). Tetric Flow ${ }^{\mathrm{TM}}$ and Admira Flow ${ }^{\mathrm{TM}}$ had a low ME (3.86 GPa and $3.29 \mathrm{GPa}$, respectively) with no significant difference between them.

Again, the high filler concentration seemed to have a great influence on the results for Grandio Flow ${ }^{\mathrm{TM}}{ }^{[6]}$. However, some authors have found an even higher ME for Grandio Flow ${ }^{\mathrm{TM}}$ than universal micro-hybrid composites ${ }^{13}$.

According to Hooke's law, stress is determined by shrinkage and the elastic modulus of the material. Polymerization shrinkage can potentially increase stress formation along the adhesive interface. 
Nevertheless, the low ME of low-viscosity composites allows them to flow during polymerization and compete with the stress development, helping maintain the marginal seal of the restoration. This compensation occurs only to a certain extent.

Another important point is that the ratio between the bound to unbound surfaces, termed the 'C-factor', is related to the shape of the prepared cavity. The relative amount of unbound resin surface determines the ability of the composite to relieve the developing stresses. A high elastic modulus shrinking restorative material confined in a high C-factor cavity will challenge and frequently destroy the bond leading to post-operative sensitivity and poor marginal quality ${ }^{2}$.

Materials with low ME, such as Admira Flow ${ }^{\mathrm{TM}}$, can be used as a liner to act as a stress relief layer. On the other hand, when selecting low-viscosity composites for restorative procedures, high polymerization shrinkage of the materials should be considered.

The use of composites, such as Grandio Flow ${ }^{\mathrm{TM}}$, with very high ME as an intermediate layer should be avoided, because of its probable incapacity to flow during conventional resin polymerization contraction when stresses are not relieved. Special attention should be given when indicating these materials for restorative procedures, especially for very small cavities with a high C-factor, since they also have high LPS. This combination of high ME and high LPS will tend to generate high contraction stresses at the adhesive interface, breaking the marginal seal. This is in agreement with other authors ${ }^{5}$. For restorative procedures using low-viscosity materials, the goal is the combination of low ME and low LPS. This will not only benefit their use as restorative materials, but also as liners minimizing the effects on the integrity of the marginal interface. In this study, this combination was found only for Tetric Flow $^{\mathrm{TM}}$.

Flowable composites comprise a rather inhomogeneous group of materials that exhibit variable composition and consequently variable mechanical and physical properties ${ }^{17}$. Clinicians must be aware of this to make proper material selection based on the specific properties and indications of each material.

The relationship between polymerization shrinkage and ME should be taken into consideration when indicating low-viscosity resin composites for restorative procedures. Composites with low shrinkage, although associated with low modulus, will tend to generate low stresses at the bonded interface. Composites with high shrinkage and high modulus will tend to produce even higher shrinkage stresses risking the integrity of the bonded interface.

In contrast, to fulfil the concept of an elastic cavity wall, the polymerization shrinkage of these materials seems irrelevant considering that the material will only be bound to one surface. Instead, the entire concept relies on the capacity of the lining materials to flow and relieve the conventional resin contraction stresses that are strongly dependent on a low ME.

\section{Conclusions}

Differences in composition directly affected LPS and ME. The prediction of a higher diluent monomer concentration favouring a high LPS was not confirmed. Tetric Flow ${ }^{\mathrm{TM}}$ presented statistically similar values to the conventional resin (Filtek $\mathrm{Z}_{250^{\mathrm{TM}}}$ ). For the ME, Grandio Flow ${ }^{\mathrm{TM}}$ presented similar values to Filtek $\mathrm{Z}_{250}{ }^{\mathrm{TM}}$, which could be attributed to a high concentration of fillers compared with the other flowables studied.

\section{References}

1. Kleverlaan CJ and Feilzer AJ. Polymerization shrinkage and contraction stress of dental resin composites. Dental Materials 2005; 21(12):1150 1157.

2. Montes MAJR, De Goes MF, Ambrosano GMB, Duarte RM and Sobrinho LC. The effect of collagen removal and the use of a low viscosity resin liner on marginal adaptation of resin composite restorations with margins in dentin. Operative Dentistry 2003; 28(4):378-387.

3. Unterbrink GL and Liedenberg WH. Flowable resin composites as "filled adhesives": literature review and clinical recommendations. Quintessence International 1999; 30(4):249-257.

4. Li Q, Jepsen S, Albers H-K and Eberhard J. Flowable materials as an intermediate layer could improve the marginal and internal adaptation of composite restorations in Class-V-cavities. Dental Materials. 2006; 22(3):250-257.

5. Cadenaro M, Marchesi G, Antoniolli F, Davidson C, Dorigo EDS and Breschi L. Flowability of composites is no guarantee for contraction stress reduction. Dental Materials 2009; 25(5):649-654.

6. Bayne SC, Thompson JY, Swift Jr EJ, Stamatiades P and Wilkerson MA. Characterization of first generation flowable composites. Journal of the American Dental Association 1998; 129(5):567-577.

7. Braga RR, Hilton TJ and Ferracane JL. Contraction stress of flowable composite materials and their efficacy as stress relieving layers. Journal of the American Dental Association 2003; 134(6):721-728.

8. Obici AC, Sinhoreti MAC, Goes MF, Consani S and Sobrinho LC. Effect of photo-activation method upon polymerization shrinkage of restorative composites. Operative Dentistry 2002; 27(1):192-198.

9. Rasband WS. ImageJ. Available from: http://rsb.info.nih.gov/ij/,19972006. Access in: July 15, 2006.

10. International Organization for Standardization - ISO. ISO 4049 Dentistry - polymer-based filling, restorative and luting materials. $3^{\text {rd }}$ ed. Geneva: ISO; 2000. p. 1-22.

11. Asmussen E and Peutzfeldt A. Influence of UEDMA, BisGMA and TEGDMA on selected mechanical properties of experimental resin composites. Dental Materials 1998; 14(1):51-56.

12. VOCO. Scientific information. Available from: http://www.voco.de/ index_en.html. Access in: Jan. 10, 2007.

13. Tagtekin DA, Yanikoglu FC, Bozkurt FO, Kologlu B and Sur H. Selected characteristics of an Ormocer and a conventional hybrid resin composite. Dental Materials 2004; 20(5):487-497.

14. Beun S, Gloriex T, Devaux J, Vreven J and Leloup G. Characterization of nanofilled compared to universal and microfilled composites. Dental Materials 2007; 23(1):51-59.

15. Walker MP, Haj-Ali R, Wang Y, Hunziker D and Williams KB. Influence of environmental conditions on dental composite flexural properties. Dental Materials 2006; 22(11):1002-1007.

16. Adabo GL, Cruz CAS, Fonseca, RG and Vaz LG. The volumetric fraction of inorganic particles and the flexural strength of composites for posterior teeth. Journal of Dentistry 2000; 31(5):353-359.

17. Stavridakis MM, Dietschi D and Krejci I. Polymerization shrinkage of flowable resin-based restorative materials. Operative Dentistry 2003; 30(1):118-128. 